\title{
Switching Control Design for Accommodating Large Step-down Disturbances in Bipedal Robot Walking
}

\author{
Hae-Won Park, Koushil Sreenath, Alireza Ramezani, and J.W. Grizzle
}

\begin{abstract}
This paper presents a feedback controller that allows MABEL, a kneed, planar bipedal robot, with $1 \mathrm{~m}$-long legs, to accommodate an abrupt $20 \mathrm{~cm}$ decrease in ground height. The robot is provided information on neither where the step down occurs, nor by how much. After the robot has stepped off a raised platform, however, the height of the platform can be estimated from the lengths of the legs and the angles of the robot's joints. A real-time control strategy is implemented that uses this on-line estimate of step-down height to switch from a baseline controller, that is designed for flat-ground walking, to a second controller, that is designed to attenuate torso oscillation resulting from the step-down disturbance. After one step, the baseline controller is re-applied. The control strategy is developed on a simplified model of the robot and then verified on a more realistic model before being evaluated experimentally. The paper concludes with experimental results showing MABEL (blindly) stepping off a $20 \mathrm{~cm}$ high platform.
\end{abstract}

\section{INTRODUCTION}

Bipedal locomotion has attracted attention for its potential ability, superior when compared to wheeled locomotion, to overcome rough terrain or environments with discontinuous supports. Existing bipedal robots, however, can only deal with small unknown variations in ground height. Ground height variations exceeding a few centimeters must be known a priori and require carefully planned maneuvers to overcome them.

Two major avenues of research are being pursued to quantify and improve the ability of a bipedal machine to walk over uneven terrain. A stochastic model of ground variation is being investigated in [1] for low-dimensional dynamical systems such as the rimless wheel and the compass bipedal walker. The mean first-passage time to the fallen absorbing state is used to assess the robustness of a gait. This metric captures the expected time that a robot can walk before falling down, measured in units of number of steps. Numerical dynamic programming is applied to a discretized representation of the dynamics to maximize the mean first-passage time. In [2], [3], [4], the gait sensitivity norm, defined as the $H_{2}$ norm of the system's state when the input is ground-height variation, is introduced to quantify the ability of a bipedal robot to handle changes in ground height. Particular attention is given to a "step-down test", where

H.-W. Park and A. Ramezani are with the Mechanical Engineering Department, University of Michigan, Ann Arbor, MI, 48109-2125, USA, parkhw@umich.edu

K. Sreenath and J. W. Grizzle are with the Control Systems Laboratory, Electrical Engineering and Computer Science Department, University of Michigan, Ann Arbor, MI 48109-2122, USA.

This work is supported in part by NSF grant ECS-909300 and in part by DARPA Contract W91CRB-11-1-0002. the ground profile consists of a flat section, followed by an abrupt decrease in height, followed again by a flat section of ground. These references use the gait sensitivity norm to assess the improvement in disturbance rejection when swingleg retraction speed at the end of the step is varied [3]. A neural network is tuned to accommodate irregular surfaces in [5]. The algorithm was tested on the robot Rabbit, whose legs are $80 \mathrm{~cm}$ long, resulting in $1.5 \mathrm{~cm}$ ground-height variations being accommodated.

While important progress is being made on walking over uneven ground, significant restrictions still remain. The experimental work in [2], [3], [4] and [5] accommodates obstacles that are less than $5 \%$ of leg length, a value that is unrealistically small when compared to common obstacles in everyday life, such as the height of steps in a building or the curb height of a sidewalk on a city street.

In this research, we propose a new control policy for the planar bipedal robot MABEL [6], which is $1 \mathrm{~m}$ at the hip and weights $65 \mathrm{~kg}$. The control policy allows MABEL to step off platforms that are at $20 \mathrm{~cm}$ high, without falling. The robot is provided information on neither where the step down occurs, nor by how much.

The remainder of the paper is organized as follows. Section II describes the general features of MABEL's morphology, and Section III summarizes a hybrid model for a walking gait. Section IV provides the design of a baseline controller reported in [7] and an initial step-down experiment reported in [8]. A switching controller is designed in Section $\mathrm{V}$ and the proposed controller is verified on a detailed simulation model in Section VI. Experimental result of the new controller are provided in Section VII. Conclusions and future directions are given in Section VIII.

\section{DESCRIPTION OF MABEL}

MABEL is a planar bipedal robot comprised of five rigid links assembled to form a torso and two legs with knees. As shown in Figure 1, the legs are terminated in point feet. All actuators are located in the torso, so that the legs are kept as light as possible. Unlike most bipedal robots, the actuated degrees of freedom of each leg do not correspond to the knee and hip angles. Instead, for each leg, a collection of cabledifferentials is used to connect two motors to the hip and knee joints in such a way that one motor controls the angle of the virtual leg consisting of the line connecting the hip to the toe, and the second motor is connected in series with a spring in order to control the length or shape of the virtual leg; see Figure 2. The reader is referred to [9], [6], [8] for more details on the transmission. 


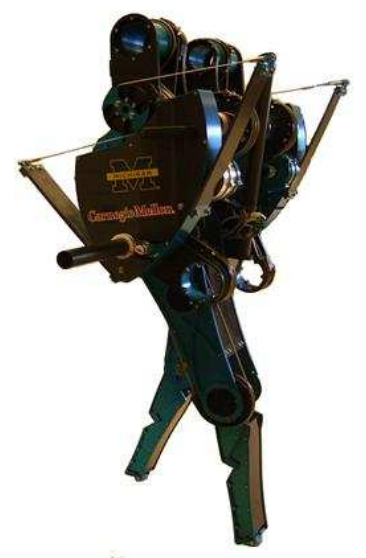

(a)

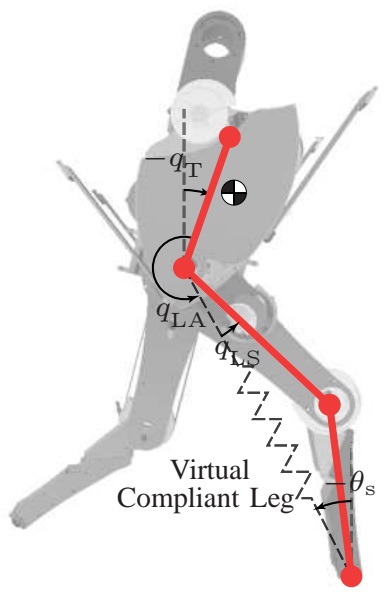

(b)
Fig. 1: (a) MABEL, an experimental testbed for bipedal locomotion. The robot is planar, with a boom providing stabilization in the frontal plane. The robot weighs $65 \mathrm{~kg}$ and is $1 \mathrm{~m}$ at the hip. (b) The virtual compliant leg created by the drivetrain through a set of differentials.

The springs in MABEL serve to isolate the reflected rotor inertia of the leg-shape motors (see Figure 2) from the impact forces at leg touchdown and to store energy in the compression phase of a walking gait, when the support leg must decelerate the downward motion of the robot's center of mass. The energy stored in the spring can then be used to redirect the center of mass upwards for the subsequent phase. These properties (shock isolation and energy storage) enhance the energy efficiency of walking and reduce the overall actuator power requirements [7].

\section{Simplified-Design Model}

Two models of MABEL have been developed and identified in [8]. This section briefly summarizes a simplified model that is appropriate for control design. In Section VI, a more detailed model appropriate for controller verification will be considered; that model includes a compliant ground contact model with a nontrivial double support phase, instead of an inelastic contact with an instantaneous double support; in addition, it accounts for the stretching that occurs in the cables used in the differentials. The simplified model will be used in Sect. V for controller design because it can be simulated twenty times faster than the detailed model.

The hybrid model consists of a continuous-time stance phase and an instantaneous double support phase. The overall dynamic model is derived with the method of Lagrange [10]. The generalized coordinates are taken as $q:=$ $\left(q_{\mathrm{LA}_{\mathrm{st}}} ; q_{\mathrm{mLS}} ; q_{\mathrm{Bsp}_{\mathrm{st}}} ; q_{\mathrm{LA}_{\mathrm{sw}}} ; q_{\mathrm{mLS}_{\mathrm{sw}}} ; q_{\mathrm{T}}\right) \in \mathcal{Q}$, where, as in Figure 1 and Figure 2, $q_{\mathrm{LA}_{\mathrm{st}}}, q_{\mathrm{mLS}_{\mathrm{st}}}$, and $q_{\mathrm{Bsp}_{\mathrm{st}}}$ are the leg angle, leg-shape motor position, and angle of the pulley $B_{\text {spring }}$ (a pulley which is connected to the free end of the spring as shown in Figure 2, and therefore corresponds to spring deflection) of the stance leg, respectively; $q_{\mathrm{LA}_{\mathrm{sw}}}$ and $q_{\mathrm{mLS}}$ are the leg angle and leg-shape motor position of the

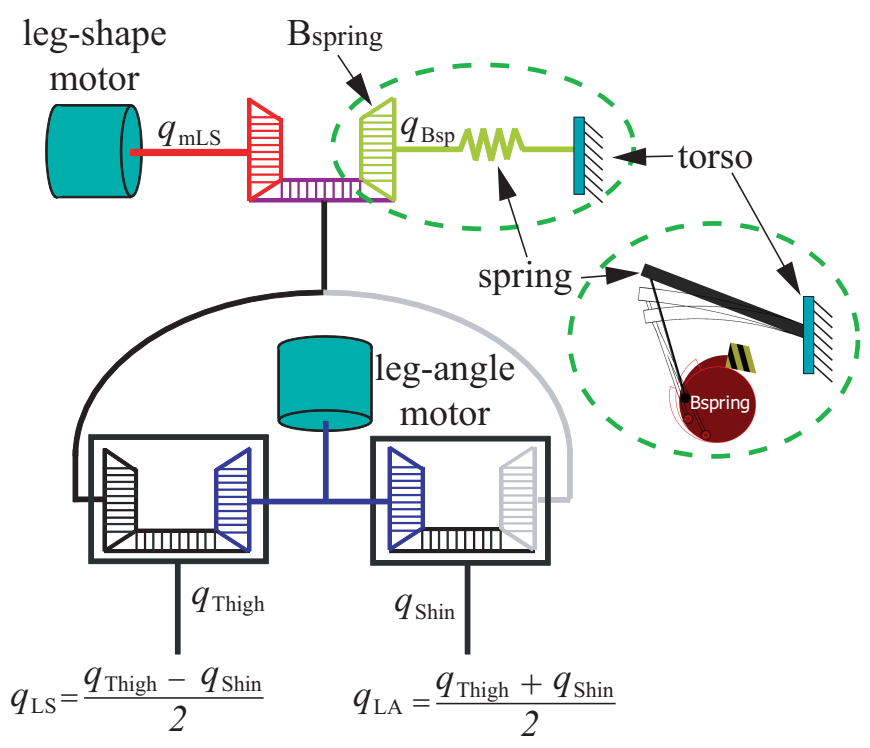

Fig. 2: MABEL's powertrain (same for each leg), all housed in the torso. Two motors and a spring are connected to the traditional hip and knee joints via three differentials, which are connected such that the actuated variables are leg angle and leg shape, see Figure 1, and so that the spring is in series with the leg-shape motor. The differentials are realized with pulleys and cables; for details, see [8].

swing leg, respectively; $q_{\mathrm{T}}$ is the angle of torso with respect to the vertical.

The state-variable form of the stance-phase dynamics, with state vector $x:=(q ; \dot{q}) \in T \mathcal{Q}$, can be expressed as,

$$
\begin{aligned}
\dot{x} & :=\left[\begin{array}{c}
\dot{q} \\
-D^{-1} H
\end{array}\right]+\left[\begin{array}{c}
0 \\
D^{-1} B
\end{array}\right] \\
& :=f(x)+g(x) u,
\end{aligned}
$$

where, $f, g$ are the drift and input vector fields, and $H:=$ $C(q, \dot{q}) \dot{q}+G(q)-B_{\text {fric }} \tau_{\text {fric }}(q, \dot{q})-B_{s p} \tau_{s p}(q, \dot{q})$. Here, $D$ is the mass-inertia matrix, $C$ is the matrix of centripetal and Coriolis terms, $G$ is the gravity vector; and the matrices $B$, $B_{\text {fric }}$, and $B_{s p}$, which are derived from the principle of virtual work, define how the actuator torques $\tau$, the joint friction torques $\tau_{\text {fric }}$, and the spring torques $\tau_{s p}$, enter the model, respectively.

An impact occurs when the swing leg touches the ground, modeled here as an inelastic contact between two rigid bodies. It is assumed that there is neither rebound nor slip at impact. Mathematically, the impact occurs when the solution $\varphi$ of (1) intersects the switching surface

$$
\mathcal{S}:=\left\{x \in T \mathcal{Q} \mid p_{\text {toe }_{\text {sw }}}^{v}(q)=0\right\},
$$

where $p_{\mathrm{toe}}^{v}(q)$ is vertical position of the swing toe. When impact occurs, the method of [11] provides a (static) map that takes the state variables just before impact to their values just after impact,

$$
x^{+}=\Delta\left(x^{-}\right) .
$$


Together, the stance-phase dynamics (1) and reset map (4) form a nonlinear system with impulse effects

$$
\left\{\begin{array}{llll}
\dot{x} & =f(x)+g(x) u & & x \notin \mathcal{S} \\
x^{+}=\Delta\left(x^{-}\right) & & x \in \mathcal{S} .
\end{array}\right.
$$

More details about the development of the impact map and the hybrid model for MABEL are presented in [7]. Control design on the basis of this hybrid model is presented next.

\section{BASEline CONTROLleR}

\section{A. Feedback design}

MABEL's baseline feedback controller is designed using method of virtual constraints [12]. The particular controller used here has been reported in [7]. The method of virtual constraints begins with the choice of outputs which depend on only configuration variables and take the form

$$
y=h(q)=h_{0}(q)-h^{d}(s(q), \alpha) .
$$

In the baseline controller, the controlled variables are

$$
h_{0}(q)=\left[\begin{array}{c}
q_{\mathrm{mLS}_{\mathrm{st}}} \\
q_{\mathrm{LA}} \\
q_{\mathrm{mLS}} \\
q_{\mathrm{T}}
\end{array}\right]
$$

and $h^{d}(s(q), \alpha)$ is a vector representing the desired evolution of the controlled variables as a function of $s(q)$, a scalar function of the configuration variables that replaces time in a standard tracking controller. The function $s(q)$ is designed to be strictly monotonically increasing over the course of a step. If a feedback can be found such that the output $y$ is driven asymptotically to zero, then the solutions of the closed-loop system asymptotically satisfy $h(q)=0$, which has the form of a holonomic constraint on a mechanical system (for more information about virtual constraints, see [13]).

In the baseline controller, the desired evolution of the controlled variables in (7) is specified by the functions $h_{\mathrm{mLS}_{\mathrm{st}}}^{d}, h_{\mathrm{LA}_{\mathrm{sw}}}^{d}, h_{\mathrm{mLS}_{\mathrm{sw}}}^{d}$, and $h_{\mathrm{T}}^{d}$, respectively, and assembled as

$$
h^{d}(s, \alpha)=\left[\begin{array}{c}
h_{\mathrm{mLS}}^{d}(s, \alpha) \\
h_{\mathrm{LA}}^{d}(s, \alpha) \\
h_{\mathrm{mLS}}^{d}(s, \alpha) \\
h_{\mathrm{T}}^{d}(s, \alpha)
\end{array}\right],
$$

where $\alpha$ is a vector of real numbers parameterizing the virtual constraints. Furthermore, $s$ is selected as

$$
s(q)= \begin{cases}\frac{\theta(q)-\theta^{+}}{\theta^{-}-\theta^{+}}, & \theta^{+}<\theta(q)<\theta^{-} \\ 1, & \theta(q) \geq \theta^{-} \\ 0, & \theta(q) \leq \theta^{+}\end{cases}
$$

where $\theta$ is the absolute angle formed by the virtual compliant leg relative to the ground, that is,

$$
\theta(q)=\pi-q_{\mathrm{LA}}{ }_{\mathrm{st}}-q_{\mathrm{T}},
$$

and $\theta^{+}$and $\theta^{-}$are the values of $\theta(q)$ at the beginning and end of a step, respectively.

How to construct the functions in $h^{d}(s, \alpha)$ from Bézier polynomials and how to choose the parameters to create a

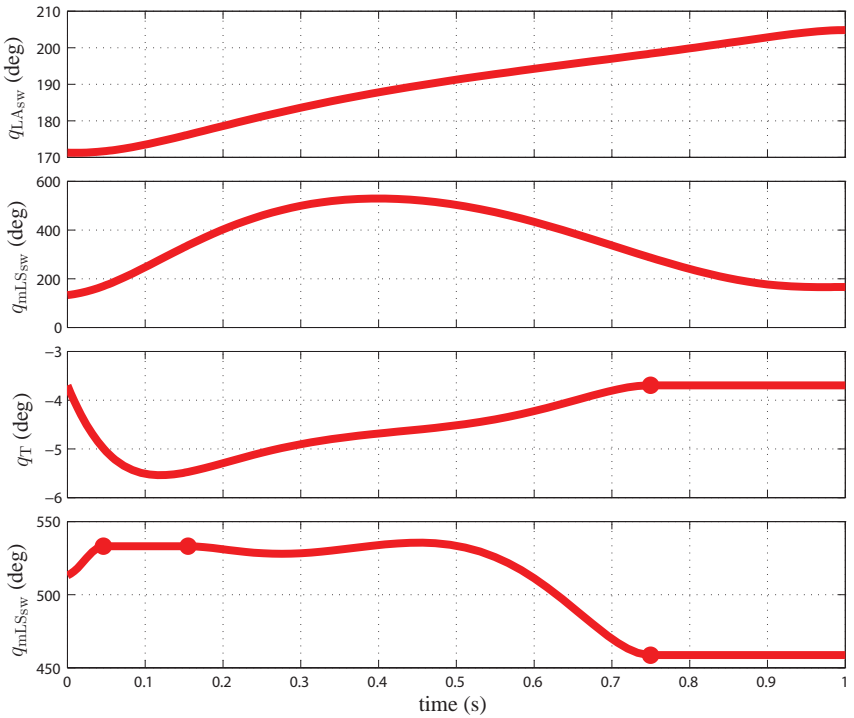

Fig. 3: Virtual constraints of the baseline controller.

periodic walking gait in the closed-loop system are explained in [12] and [7]. The main idea is to select $\alpha$ to minimize a cost function representing energy supplied by the actuators, normalized by step length, with the minimization subject to boundary conditions that specify a periodic solution, actuator magnitude and power limitations, friction limits in the ground contact model, swing-leg clearance, and desired walking speed.

In principle, the virtual constraints can be implemented on the robot by any feedback capable of driving $y$ to zero. In the experiments described below, we use the feedforwardplus-PD-controller,

$$
u^{F-P D}(x)=u^{*}(s(q), \alpha)-K_{P} y-K_{D} \dot{y},
$$

where $u^{*}(s(q), \alpha)$ is the nominal torque along the periodic orbit determined from the parameter-optimization problem when designing the virtual constraints, and $y$ is defined in (6). The asymptotic stability of the periodic orbit under this feedback law is verified on the model with a Poincare map, as explained in [12] and [7].

The above process results in the virtual constraints depicted in Figure 3. These constraints correspond to the nominal walking gait presented in [7], with average walking speed of approximately $1.0 \mathrm{~m} / \mathrm{s}$. Here, we modify the nominal virtual constraints so that the end of the swing leg at midstance can clear a $2 \mathrm{~cm}$ obstacle, allowing the robot to step onto a platform before stepping off it. Henceforth, we call this the baseline controller.

\section{B. Baseline step-down performance}

As reported in [8], using the control law (11) and the virtual constraints of Figure 3, MABEL can accommodate a 2.0 inch $(5.08 \mathrm{~cm})$ step-down disturbance. The experiment was conducted as follows. MABEL was put in motion, walking on an initially flat floor. At the end of each lap, 




Fig. 4: Experimental and data of the torso angle when stepping down from the 2.5 inch platform. The forward direction is counterclockwise, and hence corresponds to decreasing angles. The blue circles show when the swing leg impacts the ground. The torso undergoes a large oscillation as a result of the disturbance at step-down.

MABEL walked up a stair-stepped ramp ${ }^{1}$, and then stepped off the platform. The height of the platform was increased by 0.5 inches each lap until the robot fell when the platform height was increased to 2.5 inch $(6.35 \mathrm{~cm})$. MABEL fell after stepping off the 2.5 inch platform because the leg broke on the ensuing step; the video is available at [14].

Figure 4 shows the torso angle data; it can be seen that the feedback system overreacts when correcting the forwardpitching motion of the torso, causing a second, very rapid, forward-pitching motion of the torso. Because the angle of the swing leg was controlled relative to the torso, the swing leg rotated forward rapidly as well and impacted the ground with sufficient force to break the leg. Though not reported in [8], the experiment was repeated several times, with the same result, namely, a broken leg following a 2.5 inch stepdown.

Further analysis of experimental data was carried out in [8] to study the impact forces that caused the leg to break. Using the impact model of [11], the contact intensity $I_{F}$ at the leg end was estimated from the experimental data. $\mathrm{I}_{\mathrm{F}}$ has units of $\mathrm{N} \cdot \mathrm{s}$ and represents, roughly speaking, the integral of the contact force over the duration of the contact event. Figure 5 shows the estimated contact intensity when walking on flat ground and when stepping off several raised platforms. The data indicate that, upon stepping off the 2.5 inch platform, the impact intensity of the second step $47((\mathrm{~N} \cdot \mathrm{s}))$ was more than three times as intense as the impact intensity $13((\mathrm{~N} \cdot \mathrm{s}))$ of walking on flat ground.

Work presented in [8] went on to show how to design a switching controller that resulted in MABEL stepping off a 3.5 inch $8.89-\mathrm{cm}$ platform without falling. The controller

\footnotetext{
${ }^{1}$ The ramp and platform are constructed from sheets of plywood that are 0.5 inch and 1.0 inch thick. This explains the use of non-SI units.
}

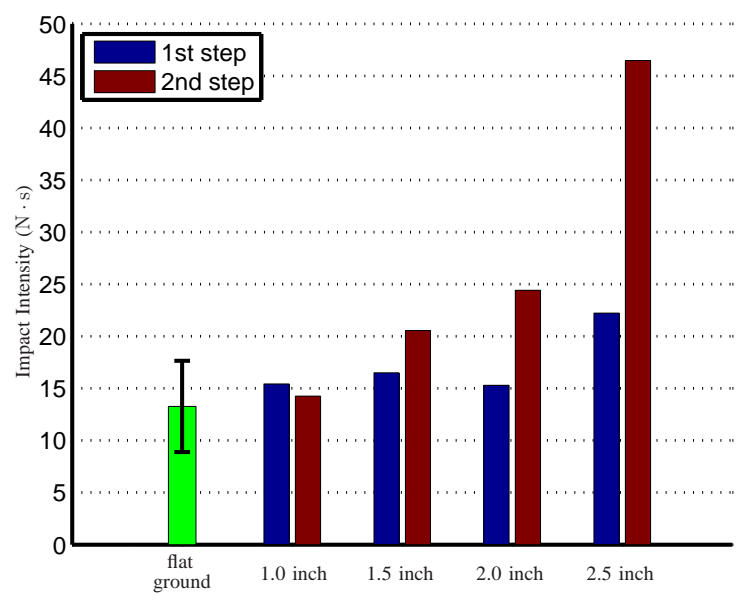

Fig. 5: Impact intensity calculated from the baseline stepdown experiments. Only the vertical component is displayed. The green bar shows the average impact intensity when walking on flat ground; the bars show \pm one standard deviation. The blue bar shows the impact intensity upon step-down, while the red bar shows impact intensity for the ensuing step. The larger impact intensity on the step following the 2.5 inch $(6.35 \mathrm{~cm})$ step-down leads to a mechanical fuse activating in the shin, which separates the leg into two pieces.

reported there used the same form of the virtual constraints shown in (6) and (8), with the virtual constraints of Figure 3 specially designed to reduce torso pitching. In the next section, a switching controller is proposed that will result in a dramatic increase in performance: MABEL stepping off an 8.0 inch $20.32 \mathrm{~cm}$ platform without falling.

\section{A Switching Controller Based on Virtual COMPLIANCE}

\section{A. Overview of basic controller}

The baseline step-down experiments showed that MABEL falls on the second step of a large step-down, and not at the moment of step-down itself. The cause of the fall is the torso pitching forward too rapidly after leg impact with the ground. To attenuate the amount of torso pitching following a large step-down, we adopt the idea of a switching controller from [15].

The height of the platform, or equivalently, the depth of the step down, can be immediately computed at impact from the lengths of the robot's legs and the angles of its joints. If the calculated height of the platform is greater than $3 \mathrm{~cm}$, the baseline controller is replaced for one step with a controller whose purpose is to attenuate pitching of the torso from the step-down disturbance. Then, at the beginning of the very next step, the baseline controller is re-applied.

\section{B. Shock absorbing controller}

The new controller, called 'shock absorbing controller', imposes virtual holonomic constraints on only three variables, $q_{\mathrm{LA}_{\mathrm{sw}}}, q_{\mathrm{mLS}_{\mathrm{sw}}}$, and $q_{\mathrm{T}}$, instead of four variables, as 
in the baseline controller. In particular, the system input corresponding to the stance motor leg shape is left free and not used for imposing a virtual constraint. Recall that this motor is in series with a physical spring in the drivetrain, as shown in Figure 2. Following an idea developed in [16] for bipedal running on MABEL, we use the torque input of this motor to create an additional virtual compliant element by defining the feedback,

$$
u_{\mathrm{mLS}_{\mathrm{st}}}(x)=-k_{\mathrm{vc}}\left(q_{\mathrm{mLS}_{\mathrm{st}}}-q_{\mathrm{mLS}_{\mathrm{vr}}}\right)-k_{\mathrm{vd}}\left(\dot{q}_{\mathrm{mLS}}\right) .
$$

This feedback essentially turns the stance leg into a shock absorber with stiffness $k_{\mathrm{vc}}$, damping $k_{\mathrm{vd}}$, and rest position $q_{\mathrm{mLS}}$. As will be seen, this method of creating a virtual compliant element serves to maintain good ground contact forces (friction cone is respected and normal component is positive) during large step-down experiments.

In principle, each of the virtual constraints for the three controlled variables $q_{\mathrm{LA}_{\mathrm{sw}}}, q_{\mathrm{mLS}_{\mathrm{sw}}}$, and $q_{T}$ can be redesigned in the shock absorbing controller. We found that the baseline virtual constraints of Figure 3 could be retained for the swing leg angle and shape; it was only necessary to redesign the virtual constraint for the torso. When redesigning the virtual constraint for the torso, the first and last coefficients of the Bézier polynomials from the baseline controller are retained; the coefficients between them, denoted hereafter by $\alpha_{\mathrm{T}}$, will be selected through optimization.

The design parameters that are to be chosen through optimization are grouped into a vector denoted

$$
\Theta:=\left[\alpha_{\mathrm{T}}, k_{\mathrm{vc}}, k_{\mathrm{vd}}, q_{\mathrm{mLS}}\right]^{T} .
$$

In general, as in [17], a suite of parameters could be precomputed for a discrete set of platform heights, such as $\{5 \mathrm{~cm}, 10 \mathrm{~cm}, 15 \mathrm{~cm}, 20 \mathrm{~cm}\}$, and the controller would then select an appropriate parameter vector, or the baseline controller, based on the estimate of platform height made at step-down. We found, however, that when the virtual compliant element in (12) is used, a step-down controller designed for $20 \mathrm{~cm}$ can accommodate step-downs of $3 \mathrm{~cm}$ to $20 \mathrm{~cm}$, and the baseline controller can accommodate variations in ground height from a $2 \mathrm{~cm}$ rise to a $3 \mathrm{~cm}$ step-down, without the controller being provided a priori information on the amount of ground height variation. This finding obviously leads to a very simple switching policy.

We next describe how the parameter vector in (13) is selected via optimization. Further details will be given in a forthcoming journal submission.

\section{Optimization process}

The optimization process is based on the simplified (control-design) model introduced in Sect. IV, with the switching surface in (3), modified to account for a change in ground height at impact,

$$
\mathcal{S}_{\mathrm{H}}:=\left\{x \mid p_{\text {toe }_{\mathrm{sw}}}^{v}=H, H \in \mathbb{R}\right\},
$$

where $H$ is the height of the platform. With this definition, the original switching surface with a platform height of zero is denoted by $\mathcal{S}_{0}$.

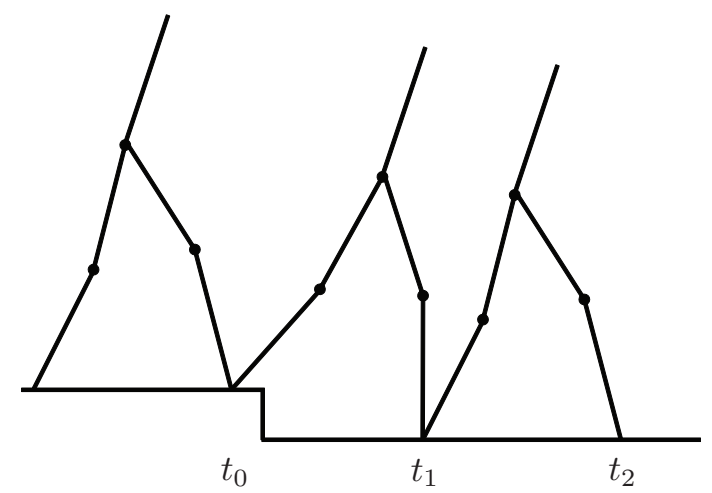

Fig. 6: Time $t_{0}$ corresponds to the end of the last step on the platform; $t_{1}$ is the end of the step-down; and $t_{2}$ is the end of the ensuing step.

Assume the robot is on the periodic orbit corresponding to the baseline controller, and hence is walking on flat ground. As in Figure 6, let $t_{0}$ be time that step-down is initiated, let $t_{1}$ be the time the swing leg impacts the ground, and let $t_{2}$ be the end of the next step, assuming it occurs. The robot is operating under the baseline controller over the interval $\left[t_{0}, t_{1}\right)$, and under the shock absorbing controller over the interval $\left[t_{1}, t_{2}\right]$. Once the step-down height $H$ is specified, $x\left(t_{1}\right)$, the state of the robot at time $t_{1}$, is known. A numerical optimization problem is posed so that the trajectory under the shock absorbing controller can be continued in such a way that the robot will not fall. In principle, the optimization could consider several steps, but only one step is considered here.

Objective: Select $\Theta$ in (13) to minimize peak-to-peak amplitude of torso oscillation as defined by,

$$
J_{\Theta}:=\max _{t \in\left[t_{1}, t_{2}\right]}\left\{q_{\mathrm{T}}(t)\right\}-\min _{t \in\left[t_{1}, t_{2}\right]}\left\{q_{\mathrm{T}}(t)\right\},
$$

where $q_{\mathrm{T}}(t)$ is trajectory of the torso angle. The cost function $J_{\Theta}$ is optimized subject to the following constraints:

1) positive horizontal swing toe position at the end of the step,

$$
p_{2}^{h}\left(t_{2}\right)>0
$$

2) maximum ratio of tangential to normal ground reaction forces experienced by the stance leg end,

$$
\max _{t \in\left[t_{1}, t_{2}\right]}\left\{\frac{F_{1}^{T}(t)}{F_{1}^{N}(t)}\right\}<\mu_{s} ;
$$

3) minimum normal ground reaction force experienced by the stance leg end,

$$
\min _{t \in\left[t_{1}, t_{2}\right]}\left\{F_{1}^{N}(t)\right\}>C \text {, for some } C>0 ;
$$

4) avoid premature impact,

$$
s\left(t_{2}\right)>1-\delta
$$

where $0<\delta \ll 1$;

5) upper bound on torso angular velocity

$$
\max _{t}\left\{\dot{q}_{\mathrm{T}}(t)\right\}<\gamma_{\dot{q}_{\mathrm{T}}} \text { for some } \gamma_{\dot{q}_{\mathrm{T}}}>0 .
$$




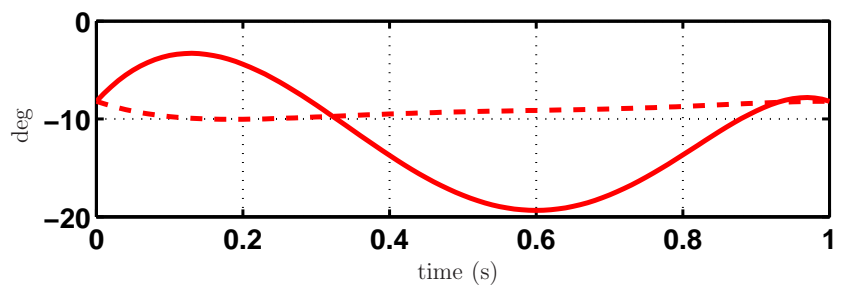

Fig. 7: Torso virtual constraints. Solid red line minimizes peak-to-peak amplitude of torso oscillation after a $20 \mathrm{~cm}$ step-down, while the dashed red line is from the baseline controller.

The optimization is conducted over a transient phase of the gait, and thus constraints are not required to impose periodicity of an orbit. We have observed that solutions to the above optimization problem tend to steer the robot so that the configuration variables at time $t_{2}$ are near their values on the periodic orbit. We conjecture that this is because the two virtual constraints for the swing leg, as well as the ending value for the torso virtual constraint, are inherited from the baseline periodic orbit.

MATLAB's constrained optimization routine fmincon is used to perform the numerical search outlined above. Setting $H=-0.2 \mathrm{~m}$, corresponding to a $20 \mathrm{~cm}$ step-down, $\delta=0.05$, $\gamma_{\dot{q}_{T}}=250 \mathrm{deg} / \mathrm{sec}, C=200 \mathrm{~N}$, and $\mu_{s}=0.5$, yields the parameter vector

$$
\begin{aligned}
\Theta= & {[0.1417, \quad-0.2338, \quad-0.7765, \quad-0.0529,2.1088,} \\
& 0.1297, \quad 2.7028]^{T} .
\end{aligned}
$$

Figure 7 shows the torso virtual constraint resulting from the optimization.

The resulting switching controller is then applied to the simplified model with a $20 \mathrm{~cm}$ step-down. The torso trajectory is shown in Figure 8. It is observed that the torso oscillates approximately $11^{\circ}$ during the step following the step-down (red solid line) and then quickly converges to its nominal trajectory. On the hand, under the the baseline controller (red dashed line), the torso noticeably overshoots when returning to the nominal lean angle. Figure 9 shows the normal ground reaction force and pulley $B_{\text {spring }}$ angle, which are important indicators of ground contact condition.

Before implementing the controller on the robot, it is evaluated on a controller validation model developed in [8]. As mentioned in Section III, the validation model takes into account more aspects of the physical robot, but it is too unwieldy for controller design when optimization is involved.

\section{Controller Verification on Detailed Model}

The model used for control design does not fully reflect experimental reality due to the following reasons: cable stretch in the robot's drivetrain; asymmetry due to the boom radius not being large enough; the simplified impact model assumes an instantaneous double support phase, whereas, in experiments, the double support phase lasts approximately $20 \mathrm{~ms}$. More details are provided in [6], [7] and [8]. By

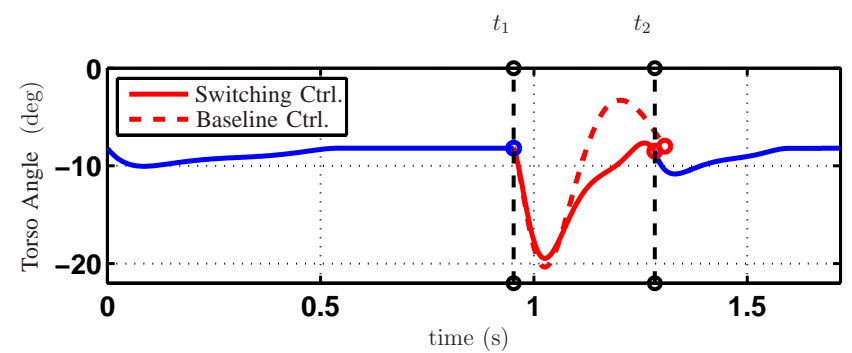

Fig. 8: Simulated torso trajectory with optimal parameter vector $\Theta$ from the simplified model for a step-down height of $20 \mathrm{~cm}$. The switching controller is shown with a solid line, while a dashed line represents the baseline controller. Red line indicates the step following step-down.

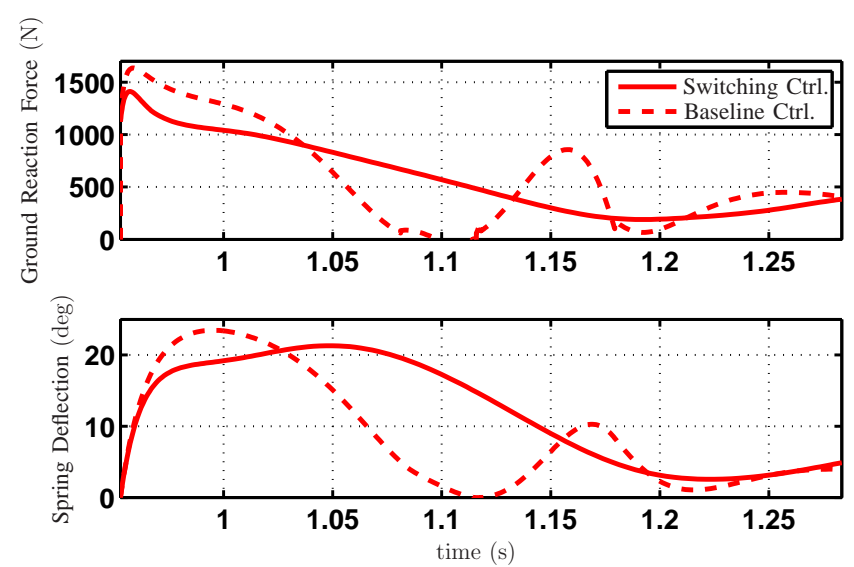

Fig. 9: Simulation data with optimal parameter vector $\Theta$ from the simplified model for a step-down height of $20 \mathrm{~cm}$. The switching controller is shown with a solid line, while a dashed line represents the baseline controller. Red line indicates the step following step-down.

representing cable stretch as a spring-damper, the boom dynamics to account for asymmetry side-to-side, and a ground model comprised of compliant ground and LuGre friction model [18], [19] into the mathematical model, the accuracy of the model is significantly improved. However, because of the complexity of this model, simulations of the detailed model take 20 times longer than the simple model developed in [7]. Hence, this model is not appropriate for optimization processes which may require thousands of simulations.

Therefore, to take only advantage of each model's strengths, low computational effort for simple model and high accuracy for detailed model, iterative controller design is conducted on the simple model first, and then the designed controller is tested on the detailed model, before implementing it on the robot.

As part of implementing the proposed controller on the detailed model, two modifications are made to account for the cable stretch which is the most critical reason for model discrepancy: (A) the coefficients of the virtual compliance in (12) are modified so that the series connection of the compliance due to the cable stretch and the virtual compliance has the effective compliance specified by the optimization 

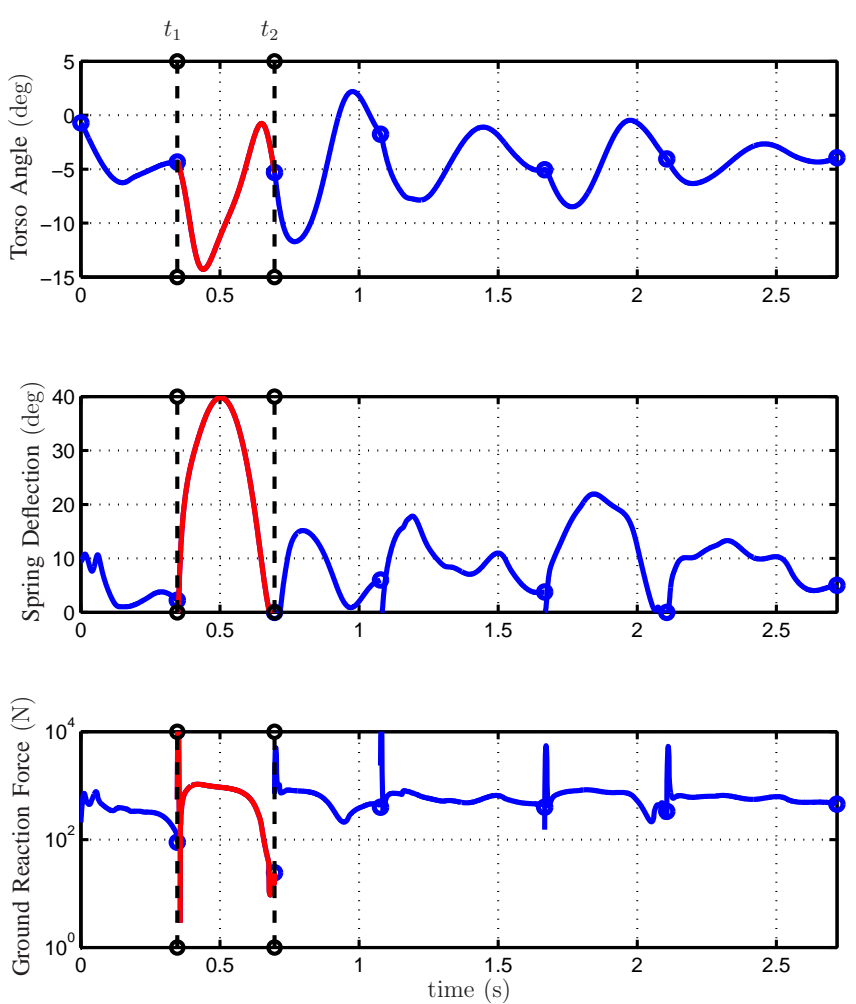

Fig. 10: Simulation data from the detailed model. Solid red line represents step following step-off. Note that ground reaction force is in log scale.

process [16]; (B) the swing leg height is increased according to the platform height calculated at impact to prevent foot scuffing; (C) in addition, a transition phase ${ }^{2}$ is added between the shock absorbing and baseline controller. With these modifications to the proposed controller, the simulation results in MABEL successfully stepping off a $20 \mathrm{~cm}$ platform.

Figure 10 shows $q_{\mathrm{T}}, q_{\mathrm{Bsp}_{\mathrm{st}}}$, and the ground reaction force on the stance toe. Despite the significant torso oscillation after step-down, the calculated impact intensity $20.1 \mathrm{~N} \cdot \mathrm{s}$ from the simulation data is less than half of the impact intensity $46.5 \mathrm{~N} \cdot \mathrm{s}$ observed in the 2.5 inch step-down under the baseline controller (see Figure 5). Furthermore, it is observed that the torso oscillation damps out rapidly over the ensuing steps and the vertical ground reaction force is positive.

\section{EXPERIMENT}

The switching controller is now evaluated on the robot. In the experiment, MABEL starts walking of a flat floor, walks up a ramp to a 7 inch $(17.78 \mathrm{~cm})$ platform and steps off, and completes a second lap before being stopped by a researcher. With the robot stopped, the platform is increased to 8 inch $(20.32 \mathrm{~cm})$. The robot was then given a push to put back in motion and stepped off the platform without falling.

Experimental data from the 8 inch step-down are shown in Figures 11 and 12. Following the step-down, the amplitude

\footnotetext{
${ }^{2}$ This will be more fully explained in an upcoming journal publication.
}
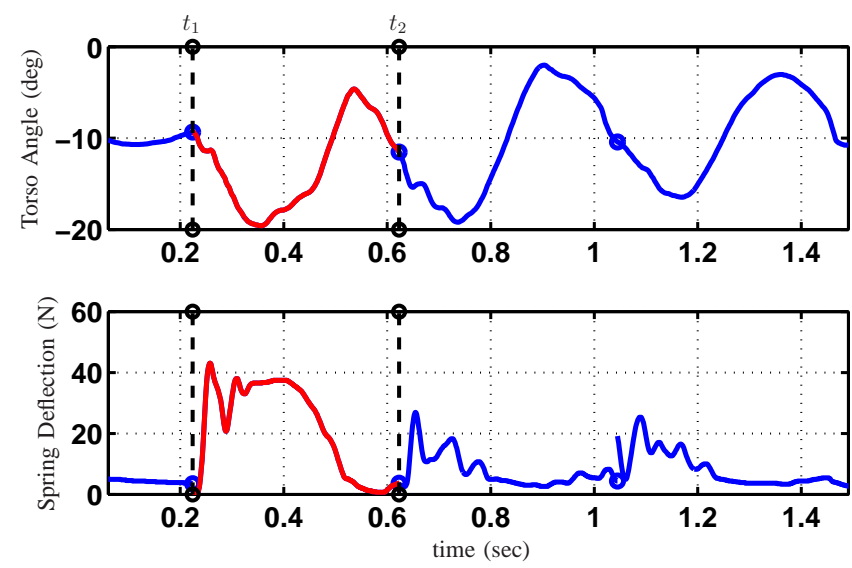

Fig. 11: Experimental data. The step-down occurs at 0.2 (s). The following step is marked as a red line.

of torso oscillation is approximately $15^{\circ}$, while the detailed model predicted a value of $13.5^{\circ}$. Remarkably, with the switching controller, the torso oscillates less after the 8 inch step-down than it does after the amplitude of 2.5 inch stepdown test when using the baseline controller, even though the platform is more than three times as high. In addition, the calculated impact intensity at the second step is $23.8 \mathrm{~N} \cdot \mathrm{s}$, which is also smaller than the value of the 2.0 inch step-down test.

The spring deflection peaks at slightly over $40^{\circ}$ just after step-down and reaches zero for a short interval of time at the end of the step, as shown in Figure 11. Two steps after the step-down event, the torso and and spring deflection are indistinguishable from steady-state walking on flat ground, showing that the gait induced by the switching controller is smoothly steered to the gait of the baseline controller.

Snapshots from video capture and a stick figure illustration of the experiment are shown in Figure 12. This data also demonstrate that the walking gait converges quickly to its steady-state characteristics following the step-down event. The video is available at [20].

\section{CONCLUSION}

A switching controller has been designed to handle blind step-downs of considerable height. Experimentation with this controller showed MABEL stepping off a $20 \mathrm{~cm}$ platform. Our next work will focus on stepping onto a platform which is higher than $5 \mathrm{~cm}$. It is conjectured that proper combination of these two step-up and step-down controllers will provide considerable robustness to uneven terrain.

\section{REFERENCES}

[1] K. Byl and R. Tedrake, "Metastable walking machines," Int. J. Rob. Res., vol. 28, pp. 1040-1064, August 2009. [Online]. Available: http://portal.acm.org/citation.cfm?id=1577179.1577185

[2] D. Hobbelen and M. Wisse, "A disturbance rejection measure for limit cycle walkers: The gait sensitivity norm," Robotics, IEEE Transactions on, vol. 23, no. 6, pp. $1213-1224$, dec. 2007.

[3] — "Swing-leg retraction for limit cycle walkers improves disturbance rejection," Robotics, IEEE Transactions on, vol. 24, no. 2, pp. $377-389$, april 2008. 


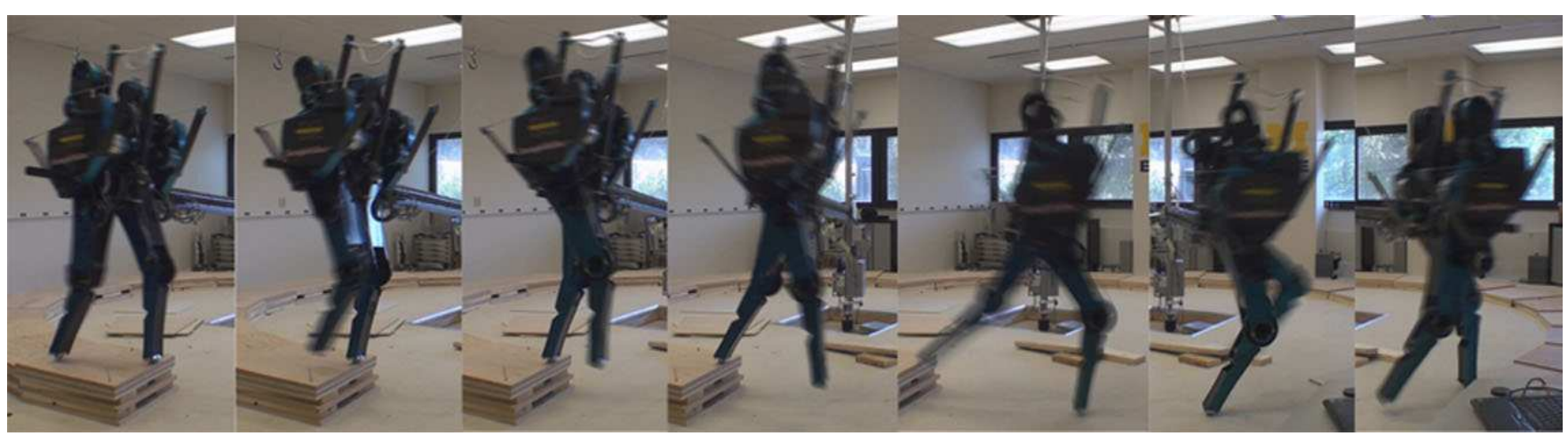

(a)

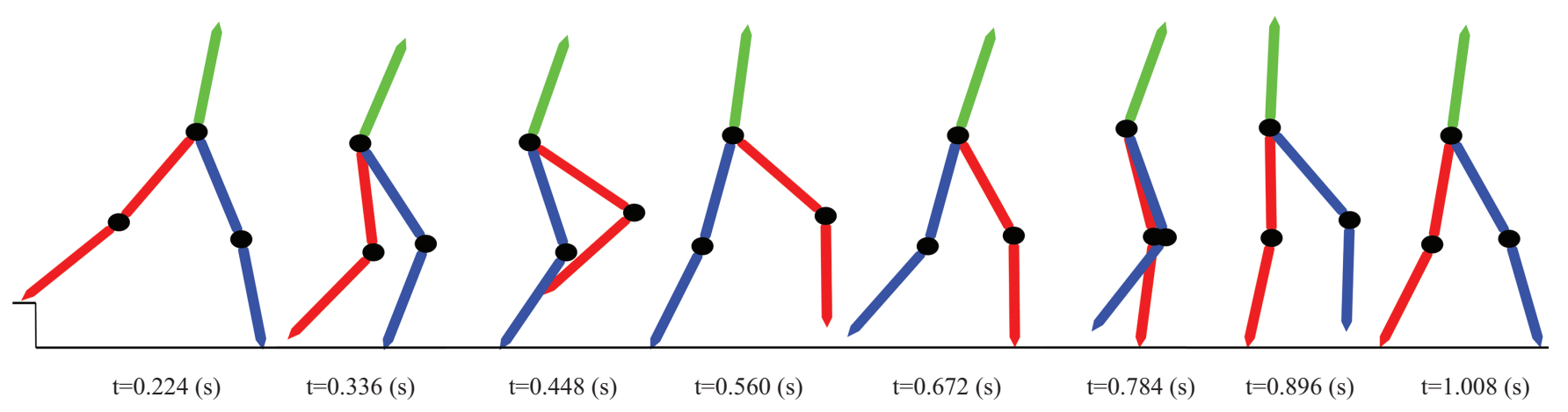

(b)

Fig. 12: Experimental data for the 8 inch step-down. Snapshots from video capture is shown in (a), while (b) shows a stick-figure illustration of the two steps following the step-down disturbance. The video is available at [20].

[4] M. Wisse, A. L. Schwab, R. Q. van der Linde, and F. C. T. van der Helm, "How to keep from falling forward: Elementary swing leg action for passive dynamic walkers," IEEE Trans. on Robotics, vol. 21, no. 3, pp. 393-401, June 2005.

[5] C. Sabourin, O. Bruneau, and G. Buche, "Control strategy for the robust dynamic walk of a biped robot," The Int. J. of Robotics Research, vol. 25, no. 9, pp. 843-860, Sept. 2006.

[6] J. Grizzle, J. Hurst, B. Morris, H.-W. Park, and K. Sreenath, "MABEL, a new robotic bipedal walker and runner," in Proc. of the American Control Conf., St. Louis, MO, USA, June 2009, pp. 2030-2036.

[7] K. Sreenath, H.-W. Park, I. Poulakakis, and J. W. Grizzle, "A compliant hybrid zero dynamics controller for stable, efficient and fast bipedal walking on MABEL," The Int. J. of Robotics Research, vol. 30, no. 9, pp. 1170-1193, Aug 2011.

[8] H.-W. Park, K. Sreenath, J. Hurst, and J. Grizzle, "Identification of a bipedal robot with a compliant drivetrain," Control Systems, IEEE, vol. 31, no. 2, pp. $63-88$, april 2011.

[9] J. W. Hurst, "The role and implementation of compliance in legged locomotion," Ph.D. dissertation, Robotics Institute, Carnegie Mellon University, Pittsburgh, PA, August 2008. [Online]. Available: http://www.ri.cmu.edu/publication_view.html?pub_id=6179.

[10] W. Khalil and E. Dombre, Modeling, Identification and Control of Robots. Bristol, PA, USA: Taylor \& Francis, Inc., 2002.

[11] Y. Hurmuzlu and D. B. Marghitu, "Rigid Body Collisions of Planar Kinematic Chains With Multiple Contact Points," The Int. J. of Robotics Research, vol. 13, no. 1, pp. 82-92, 1994.

[12] E. R. Westervelt, J. W. Grizzle, C. Chevallereau, J. H. Choi, and B. Morris, Feedback Control of Dynamic Bipedal Robot Locomotion. Taylor \& Francis/CRC Press, 2007.

[13] C. C. de Wit, "On the concept of virtual constraints as a tool for walking robot control and balancing," Аnnual Reviews in Control, vol. 28, no. 2, pp. 157-166, 2004.

[14] H.-W. Park. (2010) First attempt at walking over rough ground for bipedal robot mabel. Youtube Video. [Online]. Available: http://youtu.be/IlWIWf4daNs

[15] T. Yang, E. Westervelt, and A. Serrani, "A framework for the control of stable aperiodic walking in underactuated planar bipeds," in Proc. of the IEEE Int. Conf. on Robotics and Automation, Roma, Italy, April 2007, pp. 4661-4666.

[16] K. Sreenath, "Feedback control of a bipedal walker and runner with compliance," Ph.D. dissertation, The University of Michigan, 2011.

[17] E. R. Westervelt, G. Buche, and J. W. Grizzle, "Experimental validation of a framework for the design of controllers that induce stable walking in planar bipeds," The Int. J. of Robotics Research, vol. 24 no. 6, pp. 559-582, June 2004.

[18] C. Canudas de Wit, H. Olsson, K. Astrom, and P. Lischinsky, "A new model for control of systems with friction," IEEE Trans. on Automatic Control, vol. 40, no. 3, pp. 419-425, Mar. 1995.

[19] F. Plestan, J. Grizzle, E. Westervelt, and G. Abba, "Stable walking of a 7-DOF biped robot," IEEE Trans. on Robotics and Automation, vol. 19, no. 4, pp. 653-668, Aug. 2003.

[20] H.-W. Park. (2011) 8 inch $(20 \mathrm{~cm})$ blind step-down experiment on mabel. Youtube Video. [Online]. Available: http://youtu.be/ fFILlvO17jo 